\title{
Prevalence and Predictors of Cognitive Impairment Among Hypertensive Patients on Follow Up at Jimma University Medical Center, Jimma, Southwest Ethiopia
}

\section{(1) Tesema Etefa Birhanu1, (1) Mengistu Ayele Kassa1, (1) Bekalu Getachew1, (ㅇ Diriba Dereje22, (1) Asfaw Gerbi1}

1 Jimma University, Institute of Health Sciences, Department of Biomedical Sciences, Division of Anatomy, Jimma, Ethiopia

2Jimma University, Institute of Health Sciences, Department of Biomedical Sciences, Division of Physiology, Jimma, Ethiopia

\begin{abstract}
Objectives: Cognitive impairment is when a person has suffered recalling, learning new things, concentrating, or making decisions that affect his/her everyday life. Hypertension induces vascular alteration and lead to cognitive impairment by leading to hypoperfusion, ischemic and hemorrhagic stroke, and white matter injury.

This study aimed to determine the prevalence and predictors of cognitive impairment among hypertensive patients on follow up at Jimma University Medical centre, Jimma, Southwest Ethiopia.
\end{abstract}

Materials and Methods: Institution based cross sectional study design was employed from June 01 to July 15, 2018 among 279 hypertensive patients on follow-up at Jimma University Medical Centre chronic clinic, Jimma, Ethiopia. The collected data were cleared and entered into SPSS Version 20.0 for analysis. The association between the independent variables and the outcome variable (cognition level) was analyzed using logistic regression model. A $p$ value of $<0.05$ was considered statistically significant in the final model.

Address for Correspondence: Tesema Etefa Birhanu, Jimma University, Institute of Health Sciences, Department of Biomedical Sciences, Division of Anatomy, Jimma, Ethiopia

Phone: +251 0920152727 e-mail: tesemaetefa743@gmail.com ORCID: orcid.org/0000-0001-5820-0396

Received: 23.05.2019 Accepted: 15.08.2019

Cite this article as: Birhanu TE, Kassa MA, Getachew B, Gerbi A, Dereje D. Prevalence and Predictors of Cognitive Impairment Among Hypertensive Patients on Follow Up at Jimma University Medical Center, Jimma, Southwest Ethiopia. EJCM 2019;7(3):117-125.

DOI: 10.32596/ejcm.galenos.2019.05.031 


\begin{abstract}
Results: Out of the 279 hypertensive patients included in this study, $142(50.9 \%)$ were male and the remaining proportion was female. The mean age \pm SD of the participants was $53.15+11.544$ years with a range of 20 to 86 years. The prevalence of cognitive impairment in this study was 86 (30.8\%). Triglycerides level $>200 \mathrm{mg} /$ $\mathrm{dL}(\mathrm{AOR}=3.570,95 \% \mathrm{CI}=1.598-2.977)$ and high blood pressure Stage II HTN were significantly associated with
\end{abstract}

\section{Introduction}

Cognition states the processing of information, applying knowledge and changing preference. Cognitive function majorly includes focused attention, executive function, recall, producing and understanding language, solving problem, and making decisions ${ }^{(1)}$.

Hypertension, a chronic elevation in blood pressure exceeding $140 \mathrm{mmHg}$ systolic (SBP) or $90 \mathrm{mmHg}$ diastolic (DBP), can lead to target organs to be damaged (brain, heart and kidneys) by inducing detrimental events. Cerebral blood vessels are the main target of the deleterious effects of hypertension on the brain ${ }^{(2)}$. Hypertension causes typical alterations in small arteries and arterioles supplying the subcortical and basal ganglia white matter, resulting in small vessel disease, a major cause of lacunar strokes and cerebral hemispheric white matter damage. The resulting structural and functional cerebrovascular alterations underlie many of the neuron pathological abnormalities responsible for the cognitive deficits, including white matter damage, micro infarcts, micro bleeds, silent brain infarcts, and brain atrophy ${ }^{(3)}$. Hypertension induces vascular alteration and leads to cognitive impairment by causing hypoperfusion, ischemic and hemorrhagic stroke, and white matter injury ${ }^{(4)}$. Hypertension is also a risk factor for lowered cognitive function in persons without clinically diagnosed stroke and dementia ${ }^{(5)}$. Moreover, reduced abstract reasoning (executive dysfunction), slowing of mental processing speed and memory deficits are reported cognitive impairment $(\mathrm{AOR}=2.649,95 \% \mathrm{CI}=4.191-5.391)$.

Conclusion: Cognitive impairment was relatively common in the study population. The study revealed that, high triglyceride levels and Stage II HTN were significantly associated with cognitive impairment.

Keywords: Cognition, cognitive impairment, hypertension, MMSE, Jimma University

in association with hypertension ${ }^{(4)}$. Hypertensive elderly individuals appear to demonstrate declines in measures of global cognition ${ }^{(6)}$, including working memory(7), attention $^{(8)}$, and executive functioning ${ }^{(9)}$. A study conducted about the effects of hypertension on cognitive function with emphasis on psychomotor speed of air traffic controllers and pilots since the 1960s demonstrated reduced performance in individuals with hypertension ${ }^{(10)}$.

Cognitive deficits due to HTN in adults can be difficult to detect but can be divided into several domains including learning, memory, and attention ${ }^{(11)}$. The blood vessels in the prefrontal subcortical areas are often affected by severe HTN, which can affect the ability to make executive decisions (e.g., planning, attention, problem solving, verbal reasoning, etc. $)^{(12)}$.

Hypertension is one of recent growing public health problem in many developing countries including Ethiopia. In Ethiopia, there is no study published on cognitive impairment among hypertensive patients. Therefore, the results of this study will help health policy makers to give special considerations for cognitive impairment among hypertensive patients during designing diagnosis and management strategies. The study results will be used particularly in counseling of the prevention of the risk factors. It identifies modifiable risk factors. It also adds additional knowledge besides the existing literature. This study is important for further researchers as a baseline for study on this area. 


\section{Materials and Methods}

\section{Study Area and Period}

The study was conducted at Jimma University Medical Center (JUMC), Chronic illnesses Clinic in Jimma town. Jimma town is located $352 \mathrm{~km}$ Southwest of Addis Ababa, the capital city of Ethiopia. JUMC is one of the oldest university hospitals in Ethiopia. Currently, it is one of the teaching and referral hospitals in the Southwestern part of the country, providing services for approximately 15 million people in the catchment area, including chronic follow up for diabetes mellitus, hypertension and other chronic cases (hospital record). The study was conducted from June 01 to July 15, 2018.

The source population of this study was all adults. Hypertensive patients on follow up at JUMC chronic illnesses clinic were our target population. The study subjects were those hypertensive patients attending chronic illnesses clinic, fulfilling eligibility criteria and willing to participate. All hypertensive patients and those who had complete medical records were included. Those with a known cognitive impairment (psychiatric disorder, severe medical illnesses, and previous history of stroke) and those with visual, hearing, and speech difficulty were excluded from the current study.

\section{Sample Size and Sampling Technique}

The actual sample size was determined by using the single population proportion formula, where the following assumptions were considered: $50 \%$ prevalence, $95 \%$ confidence interval, and 5\% margin of error. Since the total population included 740 patients, we employed a correction formula, and then $10 \%$ nonresponse rate was added, which gave rise to a final sample size of 279. The participants were selected through the systematic random sampling technique after having the monthly client flow to the hospital.

\section{Data Collection Technique}

Data were collected by using an intervieweradministered structured questionnaire prepared particularly for this study, adapted from WHO STEP wise approach for chronic disease risk factor surveillance ${ }^{(13)}$. The questionnaire was prepared in English language, translated to local languages and then translated back to English to check for consistency. The questionnaire was developed based on the study objectives. The questionnaire contained socio-demographic factors, clinical variables such as blood pressure, behavioral variables and anthropometric measurements of body weight, height and waist circumference.

To measure cognitive function, the standard validated MMSE tool containing five components [orientation (10 points), registration ( 3 points), calculation (5 points), recall ( 3 points), language and drawing ( 9 points)] was used. The total score obtained for each study subject was 30 .

Chart review checklist was used to collect data concerning clinical variables (recorded laboratory results). Blood pressure was measured by using the Omron digital blood pressure measuring device. For each patient, three measurements were performed at two-minute intervals and then the average was taken as the final data for analysis ${ }^{(13)}$.

Waist circumference was measured with a flexible inelastic tape placed on the midpoint between the lower rib margin and the iliac crest in a perpendicular plane to the long axis of the body. Height and weight were measured by using a portable Stadiometer. Body mass index (BMI) was calculated by using a person's body weight in $\mathrm{kg}$ and body height in meters. The formula used was $\mathrm{BMI}=\mathrm{kg} / \mathrm{m}^{2}$ where $\mathrm{kg}$ is a person's weight in kilograms and $\mathrm{m}^{2}$ is their height in meters squared. Four data collectors (two BSc nurses and two psychiatry nurses) were involved in data collection and collection process was supervised.

\section{Operational Definitions}

- Cognitive impairment: Is when a person has trouble remembering, recall, orientation, registration, attention and calculation, language and praxis.

- Mini Mental State Examination (MMSE): Is a commonly used 30-point scale for assessing cognitive 
function in orientation, registration, attention and calculation, recall, language, and drawing. MMSE administration was performed according to existing standards ${ }^{(14)}$.

Hypertension: A person having SBP of $140 \mathrm{mmHg}$ and/or DBP of $90 \mathrm{mmHg}$ and above.

- Controlled BP = SBP 120-139 and DBP 80-89

- Stage I HTN = SBP 140-159 and DBP 90-99

- Stage II HTN $=\mathrm{SBP} \geq 160$ and $\mathrm{DBP} \geq 100$

Physical activity: According to WHO, adults and older adults are recommended to do a minimum of 150 moderate -intensity or $75 \mathrm{~min}$ vigorous intensity aerobic activity or their equivalent combination per week, and muscle strengthening activity or at least 30 minutes of moderate intensity activity on 5 days a week.

\section{Statistical Analysis}

Data were cleaned and entered into the computer using Epi-Data version 3.1 and exported to the Statistical Package for Social Science (SPSS) version 20.0 for analysis. Frequency, percentage and mean were computed for descriptive statistics. The association between the independent and dependent variables were analyzed using the logistic regression model. Bivariate analysis was done to select candidates for multivariate at $\mathrm{p}<0.25$. From the multivariate logistic regression, independent variables having a $\mathrm{p}$ value of $<0.05$ with 95\% confidence interval were declared as significantly associated with cognitive impairment. Finally, model fitness was checked through the Hosmer and Lameshow test ( $>00.05$ ). Results were organized by using frequency tables, graphs, and charts.

\section{Data quality assurance}

The following measures were taken to assure quality of data: before data collection, data collectors were trained on the objectives of the study, interviewing, on chart review contents and measurement techniques by the principal investigator for one day. The data collection instruments were pre-tested on the hypertensive patients at Shene Gebe Hospital (on 5\% of the sample size) and necessary modifications were made based on the results of the pre-test. Data were checked for completeness within 24 hours. Data cleaning and verification were done before entering them into SPSS.

\section{Ethical consideration}

The study was conducted after receiving ethical approval from Jimma University Ethical Review Board. Permission letter was obtained from Jimma University Institute of Health Ethical Review Board (No: IRB/098/2018) before data collection was started. Written informed consent was obtained from all study participants. Data obtained during the study were treated confidentially. The right to withdraw from the study was respected for participants.

\section{Results}

\section{Socio-demographic Characteristics of Study Participants}

A total of 294 hypertensive patients were enrolled into the study. Due to incomplete information on the laboratory records, 15 patients were not included in the analysis and hence the final analysis included 279 subjects. Out of 279 hypertensive patients included, 142 (50.9\%) were males. The mean age of participants was $53.15 \pm 11.544$ years with range of 20 to 86 years. Around two- third of them [178 (63.8\%)] were in age group between 40 and 59 years. Educational status of more than half of participants 153 (54.8) was grade 8 and lower (As indicated in Table 1).

\section{Behavioral Factors and Clinical Factors}

About 94 (33.7\%) respondents were Khat chewers. Regarding the status of smoking, 18 (6.5\%) were cigarette smokers. Forty-nine (17.6\%) respondents were alcohol drinkers. Most of them [237 (84.9\%)] were physically active. Around one-fourth of the respondents, 67 (24.0\%) had high triglycerides levels, 33 (11.8\%) had high cholesterol levels and $28(10.0 \%)$ had high low-density lipoprotein levels. Abnormal high-density lipoprotein levels in males and females were $50(17.9 \%)$ and $74(26.5 \%)$, respectively. The duration of diagnosis was $<5$ years in almost half of the 
respondents [137 (49.1\%)]. Above half of the respondents [148 (53\%)] were on medication for a duration of $<5$ years. Around one-fourth [69 (24.7\%)] of respondents' blood pressure values were high (both SBP and DBP). Half [140 $(50.2 \%)]$ of the participants body weights were abnormal, above twenty-five (Table 2).

\section{Predictors of cognitive impairment}

Table 3 shows the result of the bivariate and multivariate logistic regression analyses. In the bivariate analysis, socio-demographic factors such as age group between 40 and 59 years and above 60 years were significantly associated with cognitive impairment ( $\mathrm{COR}=0.524 ; 95 \%$ $\mathrm{CI}=0.199-1.384$ and $\mathrm{COR}=2.694 ; 95 \% \mathrm{CI}=0.990-7.530$, respectively). Additionally, being men $(\mathrm{COR}=0.552,95 \%$ $\mathrm{CI}=0.329-0.924)$ and educational status of grade 8 and lower and grade 9-12 group $(\mathrm{COR}=2.671 ; 95 \% \mathrm{CI}=1.364$ -

Table 1. Socio-demographic characteristics of hypertensive patients studied for cognitive impairment at follow up in chronic illnesses clinic, JUMC, 2018

\begin{tabular}{|l|l|}
\hline Variable (n=279) & Frequency, $\mathbf{n}(\%)$ Sex \\
\hline Sex & $142(50.9)$ \\
\hline \multicolumn{1}{|c|}{ Male } & $137(49.1)$ \\
\hline Female & \\
\hline $20-39$ & $22(7.9)$ \\
\hline $40-59$ & $178(63.8)$ \\
\hline $60+$ & $79(28.3)$ \\
\hline Educational status & $153(54.8)$ \\
\hline Grade 8 and lower & $60(21.5)$ \\
\hline Grade 9-12 & $66(23.7)$ \\
\hline College and above & \\
\hline Family history of HTN & $77(27.6)$ \\
\hline Yes & $202(72.4)$ \\
\hline No & $94(33.7)$ \\
\hline Khat chewing & $185(66.3)$ \\
\hline Yes & \\
\hline No & \\
\hline HTN: Hypertension & \\
\hline
\end{tabular}

5.230 and $\mathrm{COR}=0.571 ; 95 \% \mathrm{CI}=0.211-1.477)$ were significantly associated with cognitive impairment.

Among behavioral factors, Khat chewing, cigarette smoking and alcohol were significantly associated with cognitive impairment $(\mathrm{COR}=1.449,95 \% \mathrm{CI}=0.854$ 2.460, $\mathrm{COR}=1.466,95 \% \mathrm{CI}=0.548-3.921, \mathrm{COR}=0.447$, 95\% CI $=0.206-0.969$, respectively). Furthermore, physical inactivity was significantly associated with cognitive impairment $(\mathrm{COR}=2.355,95 \% \mathrm{CI}=1.207$ 4.598).

The analysis of clinical state- related factors, cholesterol level above $240 \mathrm{mg} / \mathrm{dL}$ and triglycerides above $200 \mathrm{mg} / \mathrm{dL}$ $(\mathrm{COR}=2.997,95 \% \mathrm{CI}=1.593-5.570)$ were also detected to be associated with cognitive impairment. Moreover,

Table 2. Clinical state-related factors and physical measurement characteristics of hypertensive patients studied for cognitive impairment at follow up in chronic illnesses clinic, JUMC, 2018

\begin{tabular}{|c|c|}
\hline Variables $(n=279)$ & Frequency (\%) \\
\hline \multicolumn{2}{|l|}{ Khat chewing } \\
\hline Yes & $94(33.7)$ \\
\hline No & $185(66.3)$ \\
\hline \multicolumn{2}{|l|}{ Cigarettes smoking } \\
\hline Yes & $18(6.5)$ \\
\hline No & $261(93.5)$ \\
\hline \multicolumn{2}{|l|}{ Alcohol } \\
\hline Yes & $49(17.6)$ \\
\hline No & $230(82.4)$ \\
\hline \multicolumn{2}{|l|}{ Physical activity } \\
\hline Inactive & $42(15.1)$ \\
\hline Active & 237 (84.9) \\
\hline \multicolumn{2}{|c|}{ Total cholesterol (mg/dL) } \\
\hline$<200$ & $192(68.8)$ \\
\hline $201-239$ & $54(19.4)$ \\
\hline$>240$ & $33(11.8)$ \\
\hline \multicolumn{2}{|l|}{ Triglyceride (mg/dL) } \\
\hline$<150$ & $132(47.3)$ \\
\hline $151-199$ & $80(28.7)$ \\
\hline$\geq 200$ & $67(24.0)$ \\
\hline
\end{tabular}


BMI of $18.5-24.9$ and $>25\left(\mathrm{~kg} / \mathrm{m}^{2}\right)$ was associated with cognitive impairment $\quad(\mathrm{COR}=3.181,95 \% \quad \mathrm{CI}=1.079$ 9.384, $\mathrm{COR}=1.375,95 \% \mathrm{CI}=0.089-2.337$ ). Finally, HTN stage II was found to be related to cognitive impairment $(\mathrm{COR}=2.363,95 \% \mathrm{CI}=1.252-4.459)$.

Hosmer lame show ( $p=0.732$ ), the model was fit for Hosmer-Lemeshow. Multivariable logistic regression analysis was done for all explanatory variables having $\mathrm{p}<0.25$ in the bivariate logistic regression analysis. However, on the multivariable analysis, the variables of triglycerides $\geq 200 \mathrm{mg} / \mathrm{dL}$ and HTN stage II were found to be significantly associated with cognitive impairment.

Therefore, those who had triglycerides $\geq 200 \mathrm{mg} / \mathrm{dL}$ were 3.5 times more likely to have cognitive impairment than those who had triglycerides below $150 \mathrm{mg} / \mathrm{dl}$. $(\mathrm{AOR}=3.570$, 95\% CI=1.598-2.977). Finally, those who had high blood pressure of Stage II HTN were 2.6 times more likely to have cognitive impairment than those who had controlled HTN $(\mathrm{AOR}=2.649,95 \% \mathrm{CI}=4.191-5.391)$ (Table 3).

\section{Discussion}

In this study, the prevalence of cognitive impairment in those who scored less than 24 out of 30 points by using MMSE was found to be $30.8 \%$. This is higher than the results obtained in Brazil and China, which were 23\% and $15.4 \%$, respectively ${ }^{(15,16)}$. This discrepancy might be due to poor blood pressure control, educational level and institutional based study. On the other hand, this study is less than the study conducted in Peru and Iran, $63.3 \%$ and $61.5 \%$, respectively ${ }^{(17,18)}$. These differences might be due to the differences in sample size, which was lower in our study, and due to the fact that these two studies included comorbid diseases in contrast to ours.

In our study, participants with triglycerides above $200 \mathrm{mg} / \mathrm{dL}$ were independent predictors of cognitive impairment, which was agreed by thestudies done in Egypt and China ${ }^{(19,20)}$. The possible mechanism may be due to that hypertriglyceridemia changes cerebral blood vessels by increasing the viscosity of blood and lowers cognitive function by causing $\operatorname{arteriosclerosis}^{(21)}$. Similarly, a study conducted in China suggests that higher normal concentrations of TG were significantly negatively associated with cognitive impairment ${ }^{(22)}$.

In this study, participants with high blood pressure were independent predictors of cognitive impairment, which was in line with the studies done in India, UK, Pittsburgh, Angola and Birmingham, respectively ${ }^{(23-25)}$. High blood pressure alters cerebrovascular structure and function, which leads to brain lesions such as cerebral atrophy, stroke and lacunar infarcts, diffuse white matter damage, micro infarcts and micro bleeds, and finally results in cognitive impairment. Possible molecular mechanism of this pathology may be because high blood pressure impairs the metabolism and transfer of amyloid- $\beta$ protein $(A \beta)$, accelerating cognitive impairment ${ }^{(26)}$. Damage to vascular endothelial cell function leads to a reduction in the ability of endothelial cells to regulate microvascular flow and to exert their antithrombotic and antiatherogenic effects ${ }^{(27)}$, which results in the reduction of resting cerebral blood flow, which intern causes decrease in oxygen and nutrient result in impaired $\mathrm{A} \beta$ trafficking and promoting amyloid aggregation, finally pre-neural inflammation and death lead to cognitive loss ${ }^{(28)}$.

\section{Limitations of the Study}

The cross-sectional study design does not provide evidence of a cause and effect relationship.

Lack of imaging data confines the ability to link hypertension and its causingneuropathology.

Undiagnosed mental illnesses and severe comorbid diseases might have affected the performance of the study subjects on the MMSE items, and then on the overall score.

\section{Conclusion}

Cognitive impairment was relatively common in our study population. The study revealed that triglycerides above $200 \mathrm{mg} / \mathrm{dL}$ and HTN stage II were the predictors of cognitive impairment. 
Table 3. Predictors of cognitive impairment among hypertensive patients at follow up clinic of JUMC, 2018

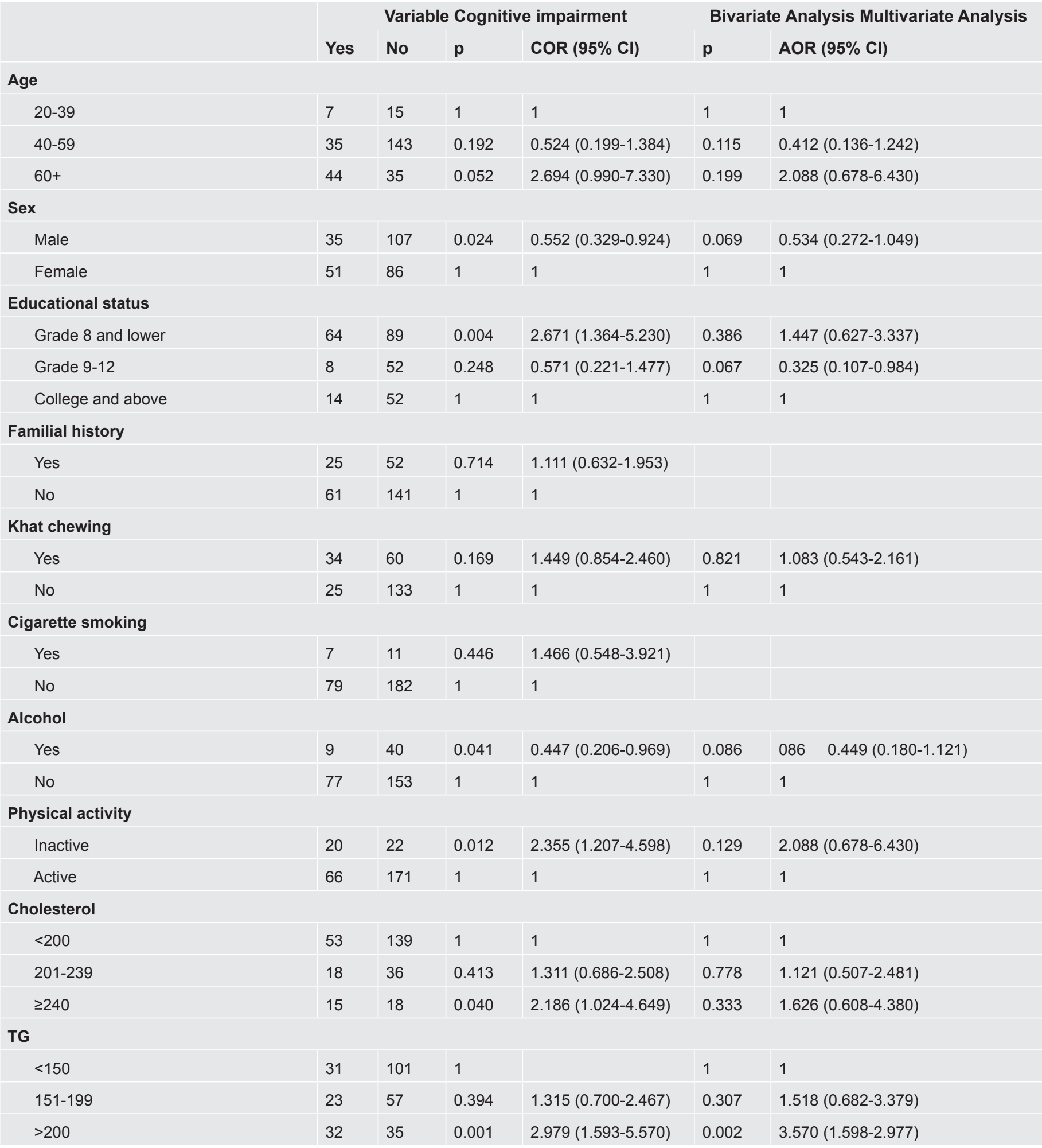


Table 3. Continued

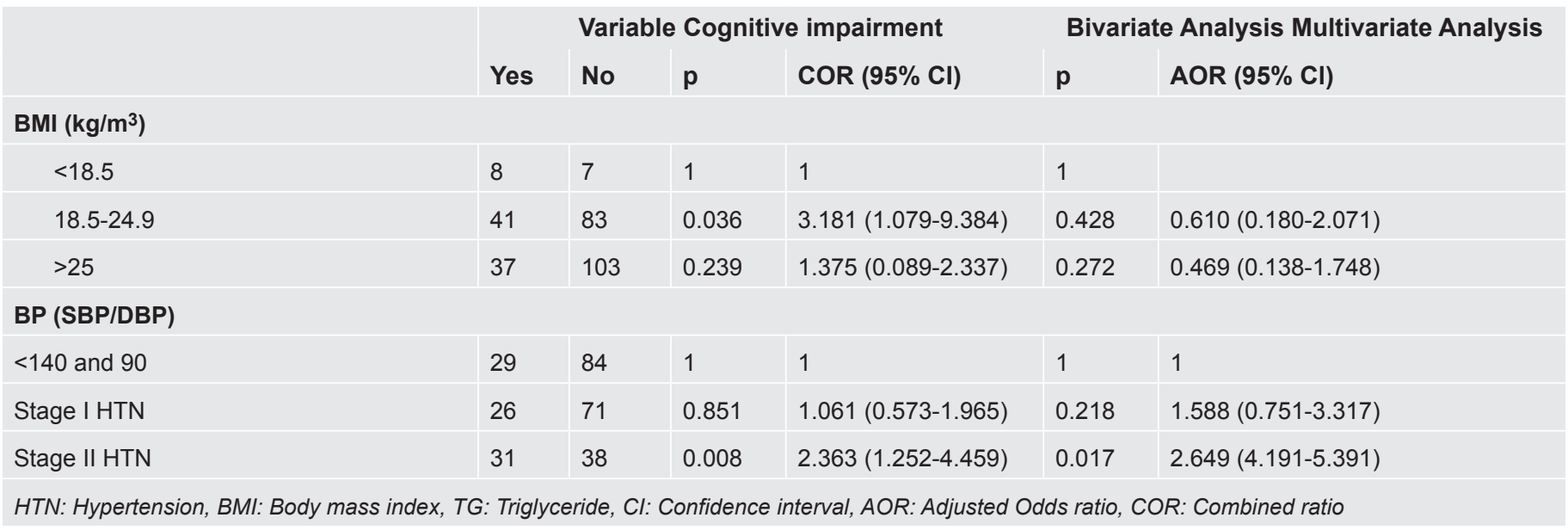

Based on our findings, the following recommendations were forwarded:

\section{Recommendation}

The JUMC should plan periodic screening of cognitive impairment among hypertensive patients to prevent further complications.

The JUMC health professionals should promote screening triglyceride and ever visit to reduce risk of cognitive impairment.

The JUMC health professionals should increase the frequency of the monitoring of patients' blood pressures in order to reduce the risk of cognitive impairment.

Moreover, the Federal Ministry of Health should promote the implementation of strategies for screening of cognitive impairment before the development of complications.

Finally, further researchers should measure clinical factors rather than chart review and they should use imaging modalities to explore the extent of cognitive impairment on hypertensive patients.

\section{Acknowledgments}

This study was funded by Jimma University Institute of Health Sciences. The authors would like to express their appreciation to Jimma University and Jimma University Medical Center for funding this research project. They would like to extend their deepest gratitude to all study participants and data collectors for their willingness and cooperation in this study.

\section{Ethics}

Ethics Committee Approval: The study was conducted after receiving Ethical approval from Jimma University Ethical Review Board. Permission Letter was obtained from the Institute of Health Sciences Ethical review board, Jimma University (No: IRB/098/2018) before data collection was started.

Informed Consent: Informed consent was taken from each study participant before data collection.

Peer-review: Internally peer-reviewed.

\section{Authorship Contributions}

Surgical and Medical Practices: T.E.B., Concept: T.E.B., B.G., D.D., Design: T.E.B., M.A.K., Data Collection or Processing: T.E.B., A.G., M.A.K., Analysis or Interpretation: T.E.B., A.G., Literature Search: T.E.B., Writing: T.E.B., B.G., D.D.

Conflict of Interest: The authors declared that there are no conflicts of interest regarding the publication of this paper. 
Financial Disclosure: This study was funded by Jimma University Institute of Health Sciences.

\section{References}

1. Jeganathan J, Seemanthini TS. Memory Impairment in Young Type 2 Diabetes Mellitus Males: An Alarming Concern! Glob J Res Anal 2014;3:172-3.

2. Perrotta, M, Lembo G, Carnevale, D. Hypertension and dementia: epidemiological and experimental evidence revealing a detrimental relationship. Int J Mol Sci 2016;17:347.

3. Shi Y, Wardlaw JM. Update on cerebral small vessel disease: a dynamic whole-brain disease. Stroke Vasc Neurol 2016;25:83-92.

4. Iadecola C, Yaffe K, Biller J, et al. Impact of hypertension on cognitive function: a scientific statement from the American Heart Association. Hypertension 2016;68:e67-94.

5. Waldstein SR. The relation of hypertension to cognitive function.Curr. Dir. Psychol Sci 2003;12:9-12.

6. Goldstein FC, Levey AI, Steenland NK. High blood pressure and cognitive decline in mild cognitive impairment. J Am Geriatr Soc 2013;61:67-73.

7. Elias MF, Dore GA, Davey A, et al. From blood pressure to physical disability: the role of cognition. Hypertension 2010;55:1360-5.

8. Hannesdottir K, Nitkunan A, Charlton RA, et al. Cognitive impairment and white matter damage in hypertension: a pilot study. ActaNeurol Scand 2009;119:261-8.

9. Waldstein SR, Brown JR, Maier KJ, et al. Diagnosis of hypertension and high blood pressure levels negatively affect cognitive function in older adults. Ann Behav Med 2005;29:174-80.

10. Elias MF, Goodell AL, Dore GA. Hypertension and cognitive functioning: a perspective in historical context. Hypertension 2012;60:260-8.

11. Si C, Ren C, Wang P, et al. Impairment in preattentive processing among patients with hypertension revealed by visual mismatch negativity. BioMed Res Int 2014;2014:945121.

12. Cha SD, Patel HP, Hains DS, et al. The effects of hypertension on cognitive function in children and adolescents. Int J Pediatr 2012;2012:891094.

13. Organization WHO. Universal health coverage on the journey towards Healthy Islands in the Pacific. Manila: WHO Regional Office for the Western Pacific. https://apps.who.int/iris/handle/10665/275483

14. Mitchell AJ, Shukla D, Ajumal HA, et al. The Mini-Mental State Examination as a diagnostic and screening test for delirium: systematic review and meta- analysis. Gen Hosp Psychiatry 2014;36:627-33.
15. Muela HC, Costa-Hong VA, Yassuda MS, et al. Hypertension severity is associated with impaired cognitive performance. J Am Heart Assoc 2017;6:e04579.

16. Wu L, He Y, Jiang B, et al. The association between the prevalence, treatment and control of hypertension and the risk of mild cognitive impairment in an elderly urban population in China. Hypertens Res 2016;39:367-75.

17. Ballesteros Rozo S. Actores individuales y colectivos asociados con la prevalencia de limitaciones funcionales del adulto mayor en Colombia. Análisis multinivel Análisismultinivel. Universidad del Rosario, 2017.

18. Sharifi F, Hedayat M, Fakhrzadeh H, et al. Hypertension and cognitive impairment: Kahrizak elderly study. Int J Gerontol 2011;5:212-6.

19. Hashem HA, Mustafa YH. Correlation between Metabolic Syndrome and Mild Cognitive Impairment. J Alzheimers Park doi: 10.4172/21610460.1000414

20. He Q, Li Q, Zhao J, et al. Relationship between plasma lipids and mild cognitive impairment in the elderly Chinese: a case-control study. Lipids Health Dis 2016;15:146.

21. Koenig W, Sund M, Ernst E, et al. Association between rheology and components of lipoproteins in human blood. Results from the MONICA project. Circulation 1992;85:2197-204.

22. Yin ZX, Shi XM, Kraus VB, et al. High normal plasma triglycerides are associated with preserved cognitive function in Chinese oldest-old. Age Ageing 2012;41:600-6.

23. Gaidhane S, Gaidhane AM, Zahiruddin QS, et al. Essential hypertension and cognitive function in elderly. Glob. J. Med. Public Health 2012;3:1-12.

24. Okusaga O, Stewart MC, Butcher I, et al. Smoking, hypercholesterolaemia and hypertension as risk factors for cognitive impairment in older adults. Age Ageing 2013;42:306-11.

25. Tsivgoulis G, Alexandrov AV, Wadley VG, et al. Association of higher diastolic blood pressure levels with cognitive impairment. Neurology 2009;73:589-95.

26. Shah NS, Vidal JS, Masaki K, et al. Midlife blood pressure, plasma $\beta$-amyloid, and the risk for Alzheimer disease: the Honolulu Asia Aging Study. Hypertension 2012;59:780-6.

27. Faraci FM. Protecting against vascular disease in brain. Am J Physiol-Heart Circ Physiol 2011;300:H1566-82.

28. Poggesi A, Pasi M, Pescini F, et al. Circulating biologic markers of endothelial dysfunction in cerebral small vessel disease: a review. J Cereb Blood Flow Metab 2016;36:72-94. 